\title{
Positional cues in serial learning: The spin-list technique
}

\author{
Michael J. Kahana, Matthew V. Mollison, and Kelly M. Addis \\ University of Pennsylvania, Philadelphia, Pennsylvania
}

\begin{abstract}
To test the hypothesis that serial learning depends largely on the encoding and retrieval of position-to-item associations, we examined whether people can learn spin lists on which starting position is randomly varied across successive learning trials. By turning positional information from a reliable cue into a source of intertrial interference, we expected learning to be greatly impaired. Contrary to this hypothesis, we found that participants were only slightly worse at serial learning under spin conditions and that this impairment reflects a substantial increase in initiation errors coupled with a small increase in intertrial forgetting. These data show that participants can effectively use nonpositional cues when positional cues are unreliable.
\end{abstract}

Ebbinghaus (1885/1913) inaugurated the laboratory study of human memory through his experiments on serial learning - the ability to reproduce a sequence of unrelated items in their order of presentation through successive study and test trials. The theoretical question that has puzzled memory scholars for more than a century since his pioneering work concerns the nature of the stored information that supports this capacity. Historically, the classic theory of serial learning was associative chaining. Chaining theory states that each item in a list is linked most strongly to its immediate neighbors (Ebbinghaus, 1885/1913; Robinson, 1932). As was noted by Ebbinghaus, "the associative threads, which hold together a remembered series, are spun not merely between each member and its immediate successor, but beyond intervening members to every member which stands to it in any close temporal relation" (p. 94). Retrieval of the first list item facilitates retrieval of the second, and the second facilitates retrieval of the third, and so forth. By somehow accessing the first (or last) item, one can chain forward (or backward) through the sequence.

Ladd and Woodworth (1911) noted that people are not limited to using just sequential relations among items to reproduce a sequence. On the basis of the strategies reported by their participants, they suggested that at least some people are able to represent positional information about the studied items and to use that positional information to facilitate recall. As it was summarized by Woodworth (1938), "Grouping, whether rhythmical or spatial, provides a blank form into which the items are inserted. . . . Remembering the list consists largely in finding the items in their places" (p. 32). According to the positional-coding hypothesis, as it came to be known, people associate each list item with a representation of the item's position in the input sequence. The first item is linked most strongly to the Position 1 marker, the second to the Position 2 marker, and so forth. During recall, items do not cue each other directly; rather, cuing with an item's position will tend to retrieve the item, and cuing with an item will tend to retrieve its position. By (somehow) sequentially cuing memory with each of the position markers, one can recall the items in either forward or backward order. Unlike chaining, which uses the items and the associations among them to support learning and recall, positional coding assumes that the participants represent the ordinal position of each item, and that they can use these positional codes to cue item recall.

Ebenholtz (1963) devised an innovative method to assess the role of positional information in serial learning. Imagine a list whose items are arranged along a circle, such that the last item is followed by the first item. In this case, there is no beginning or end of the sequence. Ebenholtz then compared two conditions: a constant starting position condition, in which the list always began with the same item (this is the standard procedure in serial learning tasks), and a varied starting position, or spin, condition, in which the list began at a different random list position on each learning trial. If associations are formed among neighboring items, as is posited by chaining theory, participants should find the spin lists only slightly more difficult to learn than standard constant starting position lists. If, however, the associations are formed between the list items and a representation of their positions in the sequence, spin lists should be extremely difficult to learn. Ebenholtz and many subsequent investigators (Battig \& Lawrence, 1967; Bowman \& Thurlow, 1963; Keppel, 1964; McManis, 1965; Saufley, 1967; Winnick \& Dornbush, 1963; Zavortink \& Keppel, 1968) consistently observed a learning deficit associated with the spin lists, but the effects were often small in magnitude. The finding that

M. J. Kahana, kahana@psych.upenn.edu 
spin lists are harder to learn has been observed for both study-test and anticipation learning methods (although the effect is larger under the study-test method; Battig \& Lawrence, 1967). Not surprisingly, the effect is reduced when the participants are informed of the circular nature of the lists (Winnick \& Dornbush, 1963). Overall, these findings have been taken as supporting some role for positional coding in serial learning.

Given the fundamental nature of serial-order memory in human experience, it is not surprising that researchers have recently re-embraced the study of serial-order memory, developing computational models to account for the many lawful phenomena documented in the experimental literature (see Lewandowsky \& Farrell, 2008, for a review). Whereas earlier computational models had assumed a central role for chaining (Lewandowsky \& Murdock, 1989), more recent accounts have largely rejected the possibility that chaining plays an important role in serial-order memory (e.g., Botvinick \& Plaut, 2006; Farrell \& Lewandowsky, 2002; Henson, Norris, Page, \& Baddeley, 1996). The recent demise of chaining accounts has been largely a consequence of findings obtained when similar or identical items occur in serial lists. In the former case, Ranschburg (1902) showed that lists with repeated items are harder to learn, but this deficit is not due to the associative interference predicted by chaining theory (Crowder, 1968; Crowder \& Melton, 1965). Rather, participants appear to have trouble correctly recalling the second of two repeated items, without exhibiting any failure at recalling the items following the repeated items, as would be predicted on the basis of chaining (e.g., Kahana \& Jacobs, 2000).

Baddeley (1968) examined serial recall of alternatingsimilarity lists; that is, the even (or odd) items were phonologically similar to one another, with the remaining items being phonologically dissimilar. For example, the alternating-similarity lists might be composed of the consonants TJBMVQD or QDMVYTJ. As a control condition, participants also studied lists consisting of phonologically dissimilar items such as HJMRYQV. Chaining theory predicts that recall of the confusable item $\mathrm{T}$ in the list TJBMVQD should lead to a competition among the nonconfusable items $\mathrm{J}, \mathrm{M}$, and $\mathrm{Q}$, thus leading to transposition errors in recall. The logic here is the same as in the studies of the Ranschburg (1902) effect, where one expects to see errors following the repeated items. In contrast, recall of the nonconfusable item J should provide an unambiguous cue for $\mathrm{B}$, although even in that case, one would expect some competition in the retrieval process, because a noisy retrieved representation of B may lead to recall of the confusable item V or D. Contrary to chaining theory, Baddeley found impaired recall only for the confusable items; recall of the nonconfusable items was unaffected by the presence of confusable items in the list. These findings were replicated in a more thorough analysis of the phenomenon reported by Henson et al. (1996).

If one accepts at face value the strong claim that chained associations are not operative in serial recall, and if one further accepts the view that most of serial learning reflects the storage and retrieval of position-to-item asso- ciations, one must wonder how people could ever learn a sequence of items when the starting position varies randomly from trial to trial, as in Ebenholtz's (1963) spinlist paradigm. Unless the participants master such lists on their first study trial, it is hard to imagine how they could be learned.

In the present study, we first sought to replicate and extend these classic spin-list studies, using modern methods that address a number of limitations of the earlier work (see the Method section). We also sought to go beyond the standard analyses of learning curves and serial position effects by analyzing the dynamics of serial recall. Specifically, we separately measured the contributions of successful initiation and successful transition to overall recall, and we decomposed the learning curve by separately looking at gains and losses of item and order information across successive study-test trials.

Comparing recall performance on spin lists with performance on control lists indicates the degree to which nonpositional cues contribute to serial learning. Three cases can be distinguished. If positional cues do not play any role in serial learning under these experimental conditions, participants should be minimally impaired in learning spin lists, and the locus of the impairment should rest primarily in the greater difficulty in correctly initiating recall under spin-list conditions (because of the interference in starting position across successive study-test trials). If, however, serial learning relies solely on positionto-item associations, and if recall of an item does not lead to recovery of its positional information, the participants should simply be unable to learn spin lists. Finally, if both associative and positional cues contribute to serial learning, one would expect to find that the participants are moderately impaired in learning spin lists and that the locus of the impairment should extend beyond recall initiation. Specifically, to the degree that consistent positional information contributes to learning, one would expect to see greater intertrial forgetting as these positional cues are put into conflict across successive spin-list trials.

\section{METHOD}

\section{Participants}

Forty-two native English speakers at Brandeis University participated for payment. Four additional participants were excluded, because they failed to learn any lists to criterion in at least one condition within the predetermined maximum number of trials (see below).

\section{Design and Procedure}

Over the course of the experiment, the participants were asked to learn 27 word lists until they could recite each list without error. The lists consisted of 7, 13, or 19 words selected randomly and without replacement from the noun subset of the Toronto Word Pool (Friendly, Franklin, Hoffman, \& Rubin, 1982). We manipulated list length to address the continuity between previous work on immediate serial recall and serial learning. In the immediate serial recall literature, most studies use short lists of $\sim 7$ items, whereas in the literature on serial learning, most researchers have tended to investigate memory for longer lists, on the order of $\sim 19$ items. If people are better able to use positional information in recalling short lists, one might expect to find a greater effect of the spin manipulation on shorter lists. 
Each list was learned using the procedure of alternating study and test trials, under either constant or varied (i.e., spin) starting position conditions. Thus, the experiment had a $3 \times 2$ within-subjects design, with three list lengths ( 7,13 , and 19) and two starting position conditions (constant and spin).

In the constant starting position condition, the participants studied lists in the usual manner of multitrial serial recall. The presentation of the list on each study trial was kept in the same order and began with the same word. The participants were asked to recall the list in the presented order. In the spin condition, the order of the list items was again kept constant. Each study trial, however, began with a randomly selected item in the list and continued in order from that item, wrapping around through all the items in the list (see Table 1 for example study-test trials). No item was used on more than one study-test trial to begin the list. The participants were asked to recall the list in the order presented on the most recent study trial.

The participants were tested in four separate sessions. An initial half-hour session was included as practice to avoid differences in learning-to-learn effects for the two conditions (Dallett, 1963) and was excluded from analysis. The practice session consisted of three lists for each starting position condition, one of each list length. The participants were informed of the list structure and starting position manipulation. Each of the three subsequent test sessions consisted of nine lists from one of the two starting position conditions - three lists of each of the three lengths $(7,13$, and 19) in random order. The order of the spin and control conditions alternated across sessions and was counterbalanced across participants.

During study, words were presented aurally at a rate of 1 word/ sec. (Each word in the pool was prerecorded by a female speaker with clear diction. Digital recordings may be obtained at memory .psych.upenn.edu.) At test, the participants were instructed to vocally recall as much of the list as they could in the order presented on the current trial without backtracking and to try to start at the beginning of the (just-presented) list. The beginning of the test phase was signaled with a row of three asterisks appearing on the screen and a 300-msec tone. Responses were recorded using a microphone for subsequent scoring of both accuracy and reaction times (RTs; due to space limitations, RT data are not reported here, but the full set of trial-by-trial data can be obtained at memory.psych.upenn .edu). The participants were given up to $1 \mathrm{~min}$ to recall as many words from the list as possible, and they indicated their completion of recall for the current trial by saying "done.” Study-test trials were continued until the participants recalled the entire list in the correct order, or until a predetermined maximum number of trials was reached (for list length 7, 7 trials; for list length 13, 13 trials; and for list length 19, 16 trials). Lists not reaching the criterion within the allotted trials were excluded from analysis; $15.9 \%$ of the spin lists and $11.8 \%$ of the control lists were not learned within the allotted number of trials. The experimenter remained present throughout all of the sessions to determine when the lists were recalled perfectly and to advance the participants to the next list.
As compared with previous studies using the spin-list technique, the present study included a number of important methodological refinements. First, the use of nonsense syllables, as was done in most early studies, adds a considerable layer of complexity, because response learning (i.e., learning the syllables themselves rather than their order) is a key determinant of performance. We used common nouns as to-be-learned items. Second, in virtually all prior studies (an exception being Battig \& Lawrence, 1967), the anticipation procedure was used. In contrast to this method, which defines the cue as the preceding item and combines learning and retrieval in a single experimental phase, our study (along with most modern studies of serial recall) uses the study-test method. In this method, study and test trials alternate, and during test trials, the participants may recall the list ad lib. Third, unlike in all previous spin-list studies, our participants were asked to recall the list vocally. This provides a more natural mode of response and avoids strategies that involve reviewing or editing previously written responses.

\section{RESULTS}

Aggregate measures of recall performance showed that the participants learned control lists more quickly than spin lists. Figure 1 shows that, with a strict positional scoring method, the mean number of trials to criterion (TTC) increased with list length $\left[F(2,82)=213.39, M S_{\mathrm{e}}=2.05\right.$, $p<.001]$ and was reliably higher in the spin than in the control condition $\left[F(1,41)=75.76, M S_{\mathrm{e}}=1.28, p<\right.$ $.001]$. The advantage of the control over the spin condition increased with list length, as is seen in a significant interaction between these two factors $\left[F(2,82)=14.16, M S_{\mathrm{e}}=\right.$ $1.17, p<.001]$. Given the literature on the importance of positional coding in immediate serial recall of short lists (see Lewandowsky \& Farrell, 2008, for a review), we had expected to find the opposite result, with spin lists causing greater difficulty in the learning of shorter lists.

In the analyses that follow, we departed from the strict positional scoring method that is common in studies of immediate serial recall. Instead, we adopted a relative order scoring method in which an item was considered to be correctly recalled if it came from the serial position immediately following that of the just-recalled item. That is, if the participants studied the list ABCDEFG and recalled the sequence ABCFG, the items B, C, and $\mathrm{G}$ would be considered correct. The first response is a special case, and one that we consider in detail in a subsequent analysis.

Table 1

Example Study and Test Trials for the Control Condition and the Spin Condition for a Hypothetical Trial With a List Length of 7

\begin{tabular}{|c|c|c|c|c|c|c|c|c|c|c|c|}
\hline \multicolumn{6}{|c|}{ Control } & \multicolumn{6}{|c|}{ Spin } \\
\hline \multicolumn{2}{|c|}{ Trial 1} & \multicolumn{2}{|c|}{ Trial 2} & \multicolumn{2}{|c|}{ Trial 3} & \multicolumn{2}{|c|}{ Trial 1} & \multicolumn{2}{|c|}{ Trial 2} & \multicolumn{2}{|c|}{ Trial 3} \\
\hline Study & Test & Study & Test & Study & Test & Study & Test & Study & Test & Study & Test \\
\hline absence & absence & absence & absence & absence & absence & absence & absence & pupil & pupil & window & window \\
\hline hollow & hollow & hollow & hollow & hollow & hollow & hollow & hollow & ribbon & ribbon & absence & absence \\
\hline pupil & & pupil & pupil & pupil & pupil & pupil & & darling & absence & hollow & hollow \\
\hline ribbon & & ribbon & window & ribbon & ribbon & ribbon & & railway & hollow & pupil & pupil \\
\hline darling & & darling & & darling & darling & darling & & window & & ribbon & ribbon \\
\hline railway & & railway & & railway & railway & railway & & absence & & darling & darling \\
\hline window & & window & & window & window & window & & hollow & & railway & railway \\
\hline
\end{tabular}

Note-The Study columns show the presented words, and the Test columns show the participant's responses. 


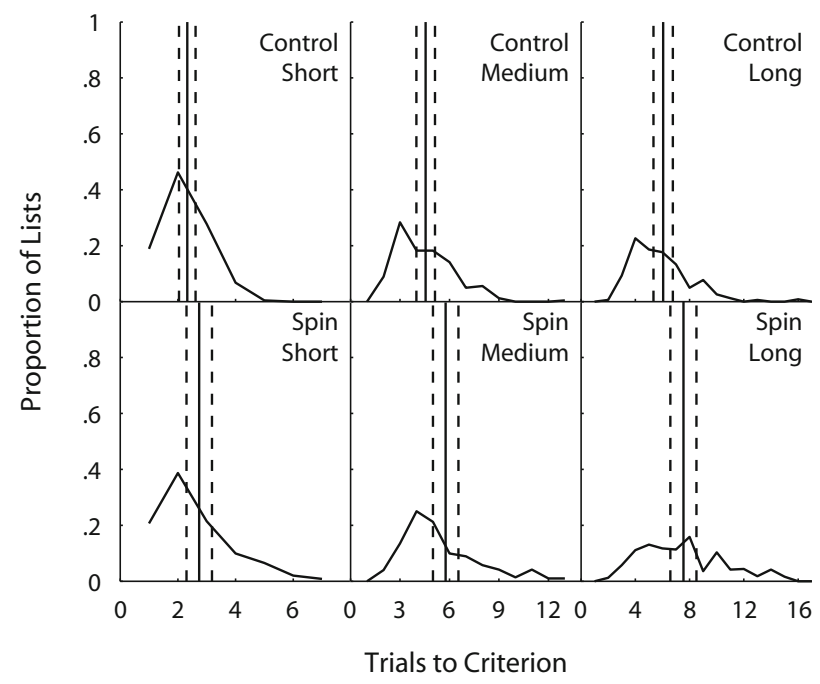

Figure 1. Distributions of study-test trials to reach a criterion of one perfect recitation. The three upper panels illustrate trials to criterion for short, medium, and long lists in the constant starting position (control) condition; the three lower panels represent data from the spin conditions. The solid vertical line in each panel indicates the estimated mean of the distribution; the dashed vertical lines indicate $95 \%$ confidence intervals around the mean. Distributions were calculated across both lists and participants using strict positional scoring.

Except where noted, we excluded the first response from our analyses of serial learning. ${ }^{1}$

Whereas strict positional scoring is appropriate for serial recall of short lists and when participants can easily indicate that they have skipped a response, with longer lists and vocal recall, positional scoring places later list items at an extreme disadvantage. For example, if a participant skipped a mid-list item and continued to correctly recall the remainder of the list, all of those subsequent recalls would be considered incorrect.

Going beyond measures of TTC, one can also observe the increased difficulty of spin lists across individual study-test trials. With the exception of the first trial, where one would not expect to see a difference between recall for spin and control lists because no change in starting position has yet occurred, most later trials exhibit significantly higher recall in the control condition. This is shown in Figure 2, in which the learning curves across the two starting position conditions for short, medium, and long lists using the relative order scoring method described above are compared. The asterisks in this figure indicate trials on which the control condition yielded significantly higher recall, as was assessed by a paired-sample $t$ test $(p<.05)$.
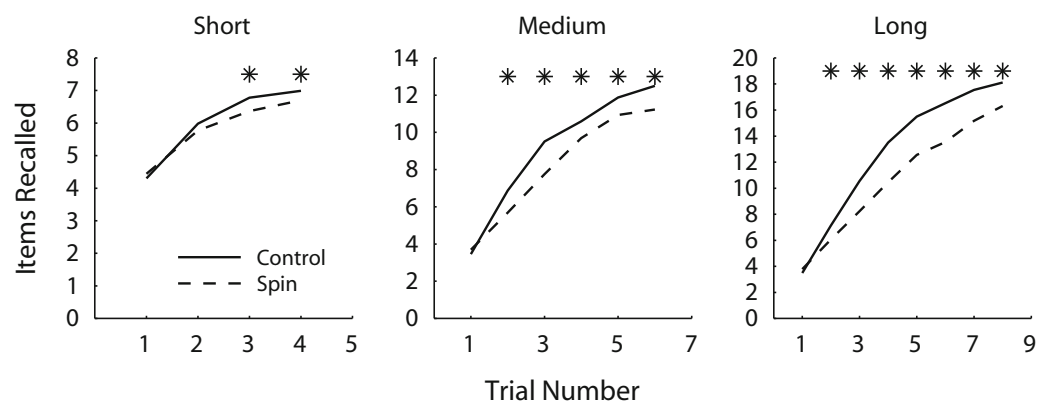

Figure 2. Learning curves for successive study-test trials. The three panels display the average number of items recalled on each test trial for short, medium, and long lists. Lists in the control condition are shown by the solid line, and spin lists are shown by the dashed line. An asterisk $(*)$ indicates a significant difference $(p<.05)$ between the control and spin conditions for that trial number. 

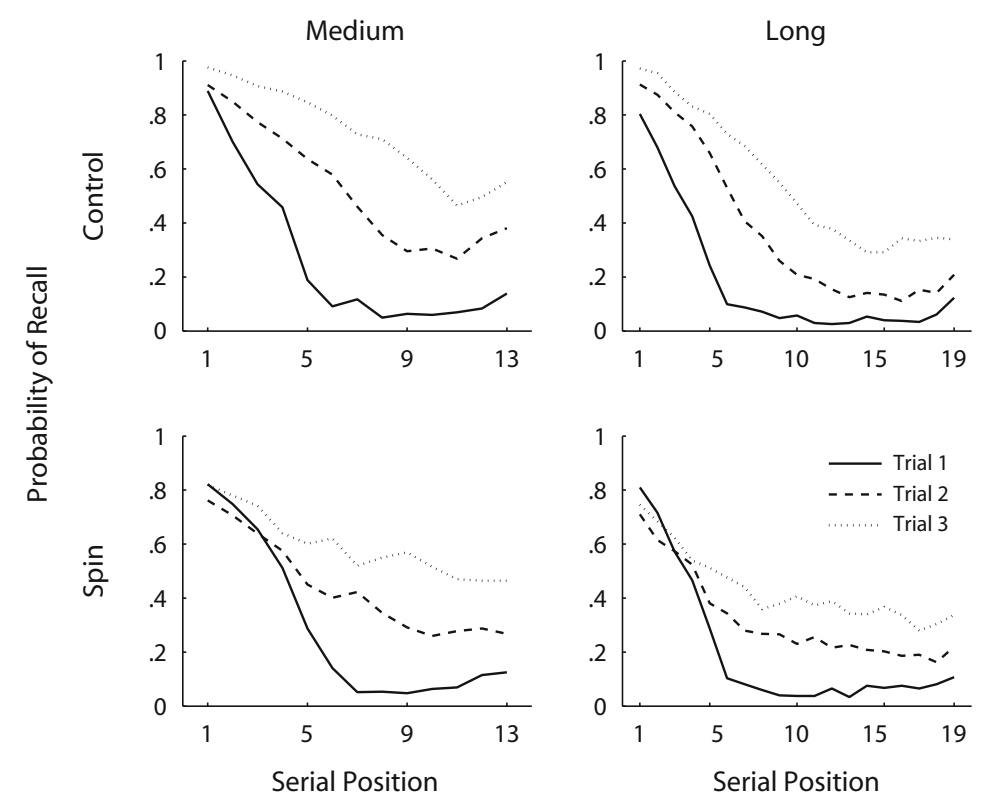

Figure 3. Serial position curves for Trials 1-3 for medium and long lists. The upper panels show data from the control conditions, and the lower panels show lists in the spin conditions.

We next compared serial position curves across successive study-test trials for spin and control lists. In this analysis of spin-list data, serial position was defined relative to the starting point of the preceding study trial, so that the starting point was always Serial Position 1. Given the high levels of recall and rapid learning of our short (list length 7) lists, we focus here and in subsequent analyses on the medium (list length 13) and long (list length 19) list conditions (contrary to our expectations, we found longer lists to exhibit the largest differences between the two conditions).

Figure 3 shows serial position curves for Trials $1-3$ for each experimental condition. As was expected, we observed greater primacy than recency, and across successive study-test trials, the overall level of recall rose, with the greatest gains accruing at the middle serial positions (e.g., Ward, 1937). For each list length, we conducted a three-way repeated measures ANOVA with starting position condition, trial number (1-3), and serial position as factors. All main effects and interactions were reliable (all $F \mathrm{~s}>3.0$, all $p \mathrm{~s}<.01)$. The interaction of starting position condition and trial suggests that differences in learning over study-test trials exist between the two starting position conditions. To further investigate this interaction, we examined the simple effects of starting position condition for Trials $1-3$ by performing a two-way repeated measures ANOVA with starting position condition and serial position as factors. As was expected, we did not observe any significant differences between spin and control lists on Trial 1 [list length $13, F(1,41)=2.59, p=.12$; list length $19, F(1,41)=2.01, p=.16]$. However, as soon as the starting position changed on Trial 2 , the serial posi- tion curve for the spin condition was significantly lower than that of the control condition [all $F \mathrm{~s}(1,41)>6.5$, all $p \mathrm{~s}<.05] .^{2}$

Although maintaining a fixed starting position across trials facilitated learning, the massive intertrial interference among positional cues in the spin condition did not prove catastrophic for the learning process. To help further understand why people have difficulty learning spin lists, we turn to an analysis of the dynamics of recall.

Our first question concerned the degree to which the participants committed recall initiation errors. In the spinlist condition, the participants were tasked with remembering the starting point of the most recent list presentation and initiating recall with that item. As such, they were very likely to suffer interference from starting positions learned on prior trials of the same list, especially on the immediately preceding trial. In the control condition, the reinforcement of the starting position on each trial should lead to a reduction in initiation errors over trials, whereas in the spin condition, the overall benefit of repetition may be offset by the large effect of interference among starting positions.

Figure 4 shows the probability of initiating recall with a list item other than the first list item. A three-way repeated measures ANOVA revealed that recall initiation errors were more likely in the spin condition $[F(1,41)=34.68$, $\left.M S_{\mathrm{e}}=0.059, p<.001\right]$ and for the longer lists $[F(1,41)=$ $\left.4.76, M S_{\mathrm{e}}=0.030, p<.05\right]$ and that the change in initiation errors with learning differed between spin and control lists $\left[F(2,82)=6.58, M S_{\mathrm{e}}=0.042, p<.01\right]$. Initiation errors decreased from Trial 1 to Trial 3 in the control condition $\left[F(2,82)=11.09, M S_{\mathrm{e}}=0.024, p<.001\right]$ but did 


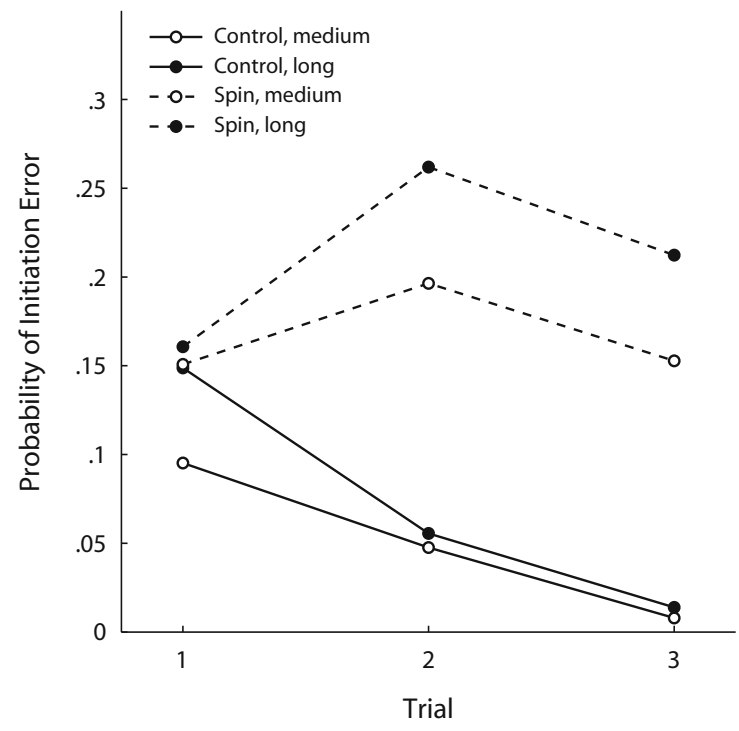

Figure 4. Probability of initiating recall with a list item other than the first list item for the first three study-test trials. Solid lines indicate data from the control conditions, and dashed lines indicate data from the spin conditions; open and filled circles indicate medium- and long-list data, respectively.

not significantly change across trials in the spin condition $\left[F(2,82)=1.35, M S_{\mathrm{e}}=0.086, p=.265\right]$ (as was assessed by separate two-way ANOVAs). If the starting position on Trial 1 is a significant source of proactive interference on Trial 2 (in the spin-list condition), one would expect that many of the initiation errors observed on Trial 2 represent cases in which the participants initiated recall with the first item presented on Trial 1. Consistent with this hypothesis, we found that among the 71 Trial 2 initiation errors, 18 were from the first serial position on Trial 1, 9 were from the second serial position, and an average of 2.6 were from each of the remaining serial positions (the remaining 5 errors were extralist intrusions).

The previous analysis shows that whereas recall initiation errors drop precipitously across study-test trials for control lists, they remain elevated throughout learning trials for spin lists. We next asked whether changes in starting position across trials (in the spin condition) also affect people's ability to learn the relative order of the list items. To measure this, we examined gains and losses on each trial. Gains were defined as items recalled in the correct relative order on trial $x$ that were either not recalled or recalled out of order on trial $x-1$. Losses were defined as items that were recalled in the correct relative order on trial $x-1$ but were subsequently not recalled or recalled out of order on trial $x$. We restricted our analysis to the transitions from Trial 1 to Trial 2 and from Trial 2 to Trial 3, averaging gains and losses over these two pairs of successive trials.

Figure 5 shows the average number of gains and losses in the control and spin conditions for medium and long lists. As was expected, gains exceeded losses in all conditions. Our critical question, however, was whether there was a significant difference in the rates of gains and losses be- tween the two starting position conditions. To assess this, we first computed ANOVAs on the rates of gains and losses as a function of list length and starting position condition.

There was no difference in the rate of gains across trials between the spin condition and the control condition $[F(1,41)<1]$. The rate of gains, however, was greater for longer than for shorter lists $\left[F(1,41)=14.04, M S_{\mathrm{e}}=\right.$ $1.275, p<.001]$. The interaction between these two factors was not significant $[F(1,41)<1]$. The rate of losses across trials was greater in the spin condition than in the control condition $\left[F(1,41)=140.65, M S_{\mathrm{e}}=0.297, p<\right.$ $.0001]$, and it was greater for longer than for shorter lists $\left[F(1,41)=10.94, M S_{\mathrm{e}}=0.416, p<.01\right]$. The interaction between these factors was not statistically significant $\left[F(1,41)=2.85, M S_{\mathrm{e}}=0.305, p=.10\right]$.

To determine whether the increased gains and losses offset one another, we computed an ANOVA on the rate of net gains (i.e., gains - losses). Here, we found slower learning (fewer net gains) in the spin condition than in the control condition $\left[F(1,41)=39.91, M S_{\mathrm{e}}=1.368, p<\right.$ $.0001]$. The difference in net gains between the two list lengths did not reach statistical significance $[F(1,41)=$ 3.03, $\left.M S_{\mathrm{e}}=1.631, p=.09\right]$. The interaction between these factors was not significant $[F(1,41)<1$, n.s.]

The preceding analyses of recall initiation and of item gains and losses suggest that people have difficulty correctly initiating recall and maintaining correctly recalled items across successive trials when the starting position varies. The first of these observations would be predicted by any associative model in which there is imperfect memory for the starting position on the most recent trial and at least some carryover of information from prior trials. Indeed, intertrial retention of associative information has been widely documented in serial recall (the so-called Hebb effect; Burgess \& Hitch, 2006; Hitch, Fastame, \& Flude, 2005; Page, Cumming, Norris, Hitch, \& McNeil, 2006), serial learning (Klein, Addis, \& Kahana, 2005), and free recall (Zaromb et al., 2006). It is less clear, however, why newly learned serial-order information is forgotten more rapidly in the spin-list condition, and why such losses are only minimally offset by an increase in item gains. One possibility is that the intertrial forgetting arises due to a loss of positional cues (i.e., the fifth item in the prior trial is no longer in the fifth presentation position on the current trial). Another possibility is that the increased losses across trials result from difficulty in correctly initiating recall. That is, if people do not succeed in keeping the starting position of the current list, and if the items correctly learned on the prior trial are not contiguous with the current trial's initial item, the increased loss of items is likely to reflect, at least in part, a lack of associative cues due to the inaccurate initiation.

To test this hypothesis, we conducted a further analysis of net gains in which we conditioned on successful recall initiation on each of the first three trials. This analysis revealed a pattern of results similar to the one that we observed in the unconditional analysis. Specifically, we found fewer net gains in the spin condition than in the control condition $\left[F(1,29)=32.52, M S_{\mathrm{e}}=0.790, p<.0001\right]$ and a greater number of net gains in the long list condition 

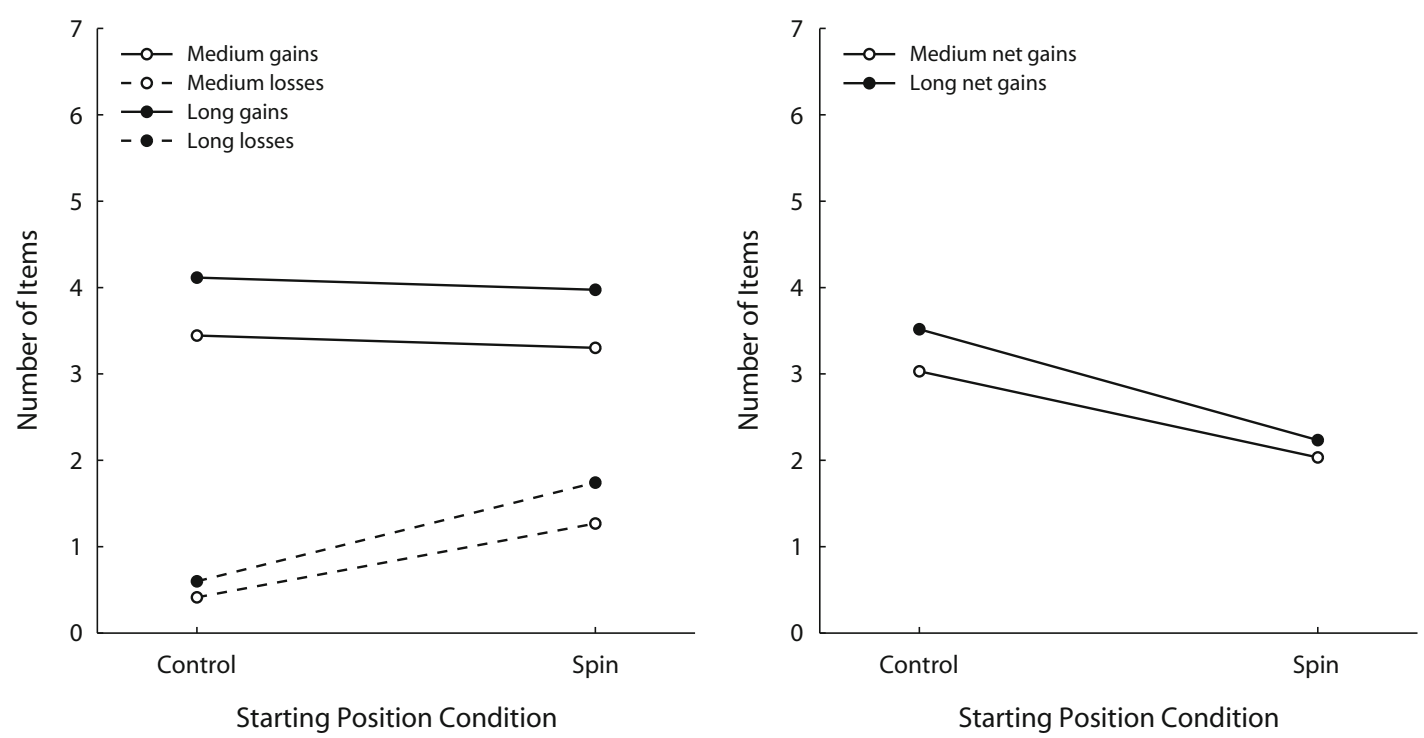

Figure 5. Gains and losses across the first three study-test trials. The left panel shows gains and losses; the right panel shows net gains. Solid lines indicate gain data, and dashed lines indicate loss data; open and filled circles indicate medium- and longlist data, respectively.

$\left[F(1,29)=21.02, M S_{\mathrm{e}}=1.413, p<.001\right]$. The interaction between these factors did not approach significance $[F(1,29)<1]$. This conditional analysis suggests that the impaired learning of spin lists is not solely a consequence of interference among the starting position items across successive trials.

\section{DISCUSSION}

Our goal in the article was to determine how the consistency of positional information across multiple study-test trials influences serial learning. This was accomplished by comparing serial learning under standard constant starting position conditions with serial learning under variable starting position, or spin, conditions. We found that people can learn short, medium, and long spin lists, but that they do so more slowly than they learn control lists. Although this difference was statistically robust, it was modest in magnitude (e.g., Cohen's $d=0.47$ for the TTC measure).

We then asked whether a more detailed analysis of the behavioral data would shed light on the factors responsible for the participants' difficulty in learning spin lists. Our first hypothesis was that the difficulty in learning spin lists stemmed from interference between varied starting positions across successive trials. Consistent with this hypothesis, the participants made many more initiation errors on spin lists than on control lists. By the third trial, the participants committed initiation errors on less than $1 \%$ of control lists; at the same point in learning, the participants committed initiation errors on over $18 \%$ of spin lists. This large difference $(d=1.03)$ is not surprising in light of the fact that participants must suffer intertrial interference from varied starting positions across successive trials.
Given that the order of all but one of the items is preserved across spin and control conditions, we hypothesized that the participants might show similar rates of item gains and losses. This analysis revealed that the participants exhibited significantly more losses in the spin condition than in the control condition and that there was no reliable difference in gains between the conditions. Thus, the net gains across trials were significantly greater in the control condition than in the spin condition. This was true even when we conditioned on lists that the participants initiated correctly. Thus, it appears that interference between the starting positions across trials cannot fully account for the participants' difficulty in learning spin lists.

The observed difficulty in learning spin lists is far smaller than what would be expected on the basis of the view that serial recall is accomplished via successive cuing with an abstract positional representation that is independent of the items themselves (e.g., Brown, Neath, \& Chater, 2007; Brown, Preece, \& Hulme, 2000; Burgess \& Hitch, 1999, 2006; Farrell \& Lewandowsky, 2002; Lewandowsky \& Farrell, 2008). Figure 6 illustrates the hypothesized encoding and retrieval processes across two successive study-test trials according to a simplified positional coding model. On Trial 2, the model predicts that each positional representation will retrieve both the appropriate item studied on Trial 2 and the inappropriate item studied on Trial 1 . The only way to completely eliminate this competition is to assume complete forgetting of the associations learned on Trial 1, in which case the participants should exhibit no learning. To the extent that the model does show transfer across trials, the transfer should be negative, since the positional representations are associated with different items on the two trials. It is thus hard to see how in such a positional model, partici- 
Study

Trial $1 \stackrel{\uparrow}{\uparrow}$
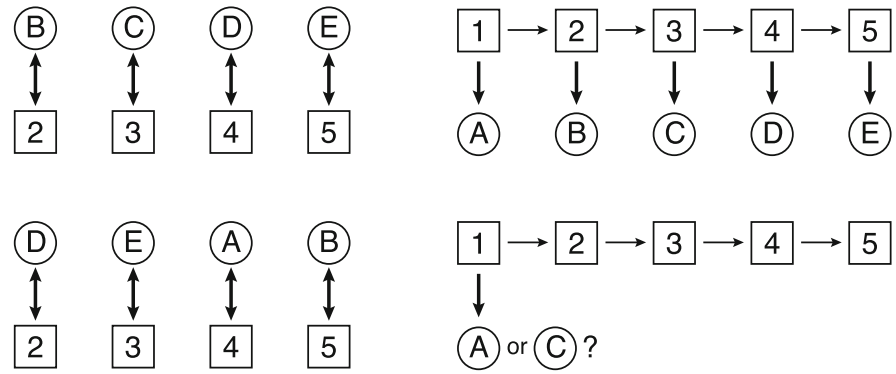

Figure 6. Illustration of the encoding and retrieval of position-to-item associations across two study-test trials in the spin-list condition.

pants could exhibit any learning under the spin conditions. One potential solution is to suppose that people learn the circular nature of the lists and develop a method by which they can map the items into a circular coordinate scheme. It remains to be seen whether a positional model could be devised to account for the relative preservation of serial learning under spin conditions.

In contrast to positional models, which predict massive interference across trials, associative chaining models (e.g., Lewandowsky \& Murdock, 1989) predict that performance should only be minimally impaired on spin trials. This is because a shift in starting position preserves the contiguity of most of the items on the list. Transfer should not be perfect, however, because each spin trial creates a break within the list where the associations are not being reinforced. Consider, for example, the list denoted by the letters ABCDEFG. If, on the second trial, the list is presented as DEFGABC, the nearest-neighbor association between $\mathrm{C}$ and $\mathrm{D}$ (and remote associations such as those between B and D) will not be reinforced. Associative chaining would also predict that participants should be impaired at recall initiation because of the interference between the start items across successive spin trials.

The original spin-list studies emerged at a time when associative chaining was the major account of serial learning. The tides have now turned, and chaining has been largely rejected as a factor underlying serial-order memory. This change in orienting views has been the result of two parallel developments: the creation of sophisticated positional coding models that can explain a very wide range of serial-order memory phenomena and the striking mispredictions of chaining theory regarding data from mixed-list phonological similarity (the so-called Sandwich effect; Henson et al.,1996) and the intralist repetition (Ranschburg, 1902) paradigms (e.g., Kahana \& Jacobs, 2000). However, the present data appear difficult to reconcile with a strict positional account. According to pure positional models (e.g., Addis, 2004), participants would not show any net gains on spin lists, contrary to the data.

In showing that people find it relatively easy to learn spin lists, the present results support a role for contiguitybased associative processes in serial recall. Several other lines of evidence also point to a role for interitem asso- ciative processes. For example, Serra and Nairne (2000) showed that in a serial reordering task, providing participants with a correct subset of items enhanced correct reordering of the remaining items. More recently, several groups have shown that following recall of an item, errors tend to be items that occurred near to the target item (not just the target position) - a pattern that mirrors that seen in free recall, where participants tend to successively recall neighboring items (Bhatarah, Ward, \& Tan, 2006, 2008; Klein et al., 2005).

The present results support a more nuanced view of serial-order memory, in which people have access to multiple sources of information and can use whichever source is best suited to solve the problem at hand. For spin lists, where inconsistent positional information will cause substantial associative interference across trials, participants would be well served to rely on contiguity-based interitem associations to facilitate learning. In contrast, under conditions of intraserial repetition or high levels of interitem similarity, learning position-to-item associations and using those associations to cue recall could enable the participants to overcome the massive interference produced by interitem associations. With long lists, positional cues are unlikely to support performance by themselves, because there is likely an upper limit on the number of discriminable positional representations. In this case, positional cues may help to identify items in terms of the relevant part of the list from which they came (e.g., Hintzman, Block, \& Summers, 1973) but not their precise serial position. These results suggest that serial learning is a complex process that reflects the storage, retention, and retrieval of multiple sources of information, including contiguity-based associations and positional associations. In addition, it is likely that serial learning is also influenced by the development of hierarchical associations (Johnson, 1972; Martin \& Noreen, 1974; Murdock, 1995), contextual associations (Howard \& Kahana, 2002; Polyn, Norman, \& Kahana, 2009; Sederberg, Howard, \& Kahana, 2008), and other sources of information yet to be uncovered. We see the spin-list technique as a particularly valuable tool in the study of serial-order memory because it transforms positional cues - arguably the most widely studied aspect of serial-order memory-from a reliable 
source of information into a source of massive intertrial interference.

\section{AUTHOR NOTE}

The authors acknowledge support from National Institutes of Health Grant MH55687 and the Dana Foundation. We are grateful to Jonathan Peelle for his help in early stages of this project. Correspondence concerning this article should be addressed to M. J. Kahana, Department of Psychology, University of Pennsylvania, 3401 Walnut St., Suite 303C, Philadelphia, PA 19103 (e-mail: kahana@psych.upenn.edu).

\section{REFERENCES}

ADDIs, K. M. (2004). Constraining models of serial learning. Unpublished doctoral dissertation, Brandeis University.

AdDis, K. M., \& Kahana, M. J. (2004). Decomposing serial learning: What is missing from the learning curve? Psychonomic Bulletin \& Review, 11, 118-124.

Baddeley, A. D. (1968). Prior recall of newly learned items and the recency effect in free recall. Canadian Journal of Psychology, 22 157-163.

Battig, W. F., \& Lawrence, P. S. (1967). The greater sensitivity of the serial recall than anticipation procedure to variations in serial order. Journal of Experimental Psychology, 73, 172-178.

Bhatarah, P., Ward, G., \& TAN, L. (2006). Examining the relationship between free recall and immediate serial recall: The effect of concurrent task performance. Journal of Experimental Psychology: Learning, Memory, \& Cognition, 32, 215-229.

Bhatarah, P., Ward, G., \& TAN, L. (2008). Examining the relationship between free recall and immediate serial recall: The serial nature of recall and the effect of test expectancy. Memory \& Cognition, 36, 20-34.

Botvinick, M. M., \& Plaut, D. C. (2006). Short-term memory for serial order: A recurrent neural network model. Psychological Review, 113, 201-233.

Bowman, R. E., \& ThurLow, W. R. (1963). Determinants of the effect of position in serial learning. American Journal of Psychology, 76 436-445.

Brown, G. D. A., Neath, I., \& Chater, N. (2007). A temporal ratio model of memory. Psychological Review, 114, 539-576.

Brown, G. D. A., Preece, T., \& Hulme, C. (2000). Oscillator-based memory for serial order. Psychological Review, 107, 127-181.

Burgess, N., \& Hitch, G. J. (1999). Memory for serial order: A network model of the phonological loop and its timing. Psychological Review, 106, 551-581.

Burgess, N., \& Hitch, G. J. (2006). A revised model of short-term memory and long-term learning of verbal sequences. Journal of Memory \& Language, $\mathbf{5 5}, 627-652$

CROWDER, R. G. (1968). Intraserial repetition effects in immediate memory. Journal of Verbal Learning \& Verbal Behavior, 7, 446-451.

Crowder, R. G., \& Melton, A. W. (1965). The Ranschburg phenomenon: Failures of immediate recall correlated with repetition of elements within a stimulus. Psychonomic Science, 2, 295-296.

Dallett, K. M. (1963). Practice effects in free and ordered recall. Journal of Experimental Psychology, 66, 65-71.

Ebinghaus, H. (1913). On memory: A contribution to experimental psychology. New York: Teachers College, Columbia University. (Original work published 1885)

Ebenholtz, S. M. (1963). Serial learning: Position learning and sequential associations. Journal of Experimental Psychology, 66, 353-362.

FARRELl, S., \& LEWANDOWSKY, S. (2002). An endogenous distributed model of ordering in serial recall. Psychonomic Bulletin \& Review, 9, 59-79

Friendly, M., Franklin, P. E., Hoffman, D., \& Rubin, D. C. (1982). The Toronto Word Pool: Norms for imagery, concreteness, orthographic variables, and grammatical usage for 1,080 words. Behavior Research Methods \& Instrumentation, 14, 375-399.

Golomb, J. D., Peelle, J. E., Addis, K. M., Kahana, M. J., \& WingFIELD, A. (2008). Effects of adult aging on utilization of temporal and semantic associations during free and serial recall. Memory \& Cognition, 36, 947-956.
Henson, R. N. A., Norris, D. G., Page, M. P. A., \& Baddeley, A. D (1996). Unchained memory: Error patterns rule out chaining models of immediate serial recall. Quarterly Journal of Experimental Psychology, 49A, 80-115.

Hintzman, D. L., Block, R. A., \& Summers, J. J. (1973). Contextual associations and memory for serial position. Journal of Experimental Psychology, 97, 220-229.

Hitch, G. J., Fastame, M. C., \& Flude, B. (2005). How is the serial order of a verbal sequence coded? Some comparisons between models. Memory, 13, 247-258.

Howard, M. W., \& Kahana, M. J. (2002). A distributed representation of temporal context. Journal of Mathematical Psychology, 46, 269-299.

JoHNSON, N. F. (1972). Organization and the concept of a memory code In A. W. Melton \& E. Martin (Eds.), Coding processes in human memory (pp. 125-159). Washington, DC: Winston.

KaHANA, M. J., \& JACOBS, J. (2000). Interresponse times in serial recall Effects of intraserial repetition. Journal of Experimental Psychology: Learning, Memory, \& Cognition, 26, 1188-1197.

KEPPEL, G. (1964). Retroactive inhibition of serial lists as a function of the presence or absence of positional cues. Journal of Verbal Learning \& Verbal Behavior, 3, 511-517.

Klein, K. A., AdDis, K. M., \& Kahana, M. J. (2005). A comparative analysis of serial and free recall. Memory \& Cognition, 33, 833-839.

LADD, G. T., \& WoODWORTH, R. S. (1911). Elements of physiological psychology: A treatise of the activities and nature of the mind from the physical and experimental point of view. New York: Scribner.

LEWANDOWSKY, S., \& FARRELl, S. (2008). Short-term memory: New data and a model. In B. H. Ross (Ed.), The psychology of learning and motivation (Vol. 49, pp. 1-48). San Diego: Academic Press.

Lewandowsky, S., \& Murdock, B. B., JR. (1989). Memory for serial order. Psychological Review, 96, 25-57.

Martin, E., \& NoreEn, D. L. (1974). Serial learning: Identification of subjective subsequences. Cognitive Psychology, 6, 421-435.

MCMANIS, D. L. (1965). Relative errors with three lengths of serial list. Psychological Reports, 16(3, Pt. 2), 1086-1088.

MurDock, B. B.[, JR.] (1995). Developing TODAM: Three models for serial-order information. Memory \& Cognition, 23, 631-645.

Nyberg, L., Sandblom, J., Jones, S., Neely, A. S., Petersson, K. M., INGVAR, M., \& BäCKMAN, L. (2003). Neural correlates of trainingrelated memory improvement in adulthood and aging. Proceedings of the National Academy of Sciences, 100, 13728-13733.

Page, M. P. A., Cumming, N., Norris, D., Hitch, G. J., \& McNeil, A. M. (2006). Repetition learning in the immediate serial recall of visual and auditory materials. Journal of Experimental Psychology: Learning, Memory, \& Cognition, 32, 716-733.

Polyn, S. M., Norman, K. A., \& Kahana, M. J. (2009). A context maintenance and retrieval model of organizational processes in free recall. Psychological Review, 116, 129-156.

RANSCHBURG, P. (1902). Über Hemmung gleichzeitiger Reizwirkungen. Zeitschrift für Psychologie \& Physiologie der Sinnesorgane, 30, 39-86.

Robinson, E. S. (1932). Association theory to-day: An essay in systematic psychology. New York: Century.

SAUfLEY, W. H., JR. (1967). An analysis of cues in serial learning. Journal of Experimental Psychology, 74, 414-419.

Sederberg, P. B., Howard, M. W., \& Kahana, M. J. (2008). A contextbased theory of recency and contiguity in free recall. Psychological Review, 115, 893-912.

SerRa, M., \& NAIRnE, J. S. (2000). Part-set cuing of order information: Implications for associative theories of serial order memory. Memory \& Cognition, 28, 847-855.

WARD, L. B. (1937). Reminiscence and rote learning. Psychological Monographs, 49, 64.

Winnick, W. A., \& Dornbush, R. L. (1963). Role of positional cues in serial rote learning. Journal of Experimental Psychology, 66, $419-421$.

Woodworth, R. S. (1938). Experimental psychology. New York: Holt. Zaromb, F. M., Howard, M. W., Dolan, E. D., Sirotin, Y. B., Tully, M., Wingfield, A., \& Kahana, M. J. (2006). Temporal associations and prior-list intrusions in free recall. Journal of Experimental Psychology: Learning, Memory, \& Cognition, 32, 792-804. 
ZAVORTINK, B., \& KePPEL, G. (1968). Retroactive inhibition of interitem associations for serial lists with constant or varied starting positions. Journal of Verbal Learning \& Verbal Behavior, 7, 771-775.

\section{NOTES}

1. The relative order scoring method that we used here is stricter than that used in a number of previous studies in which an item was considered correct if it appeared later in the list than the previously recalled item (Addis \& Kahana, 2004; Golomb, Peelle, Addis, Kahana, \& Wingfield, 2008; Klein, Addis, \& Kahana, 2005; Nyberg et al., 2003).
2. As can be seen in Figure 3, varying starting position resulted in a flattening of the serial position curves over study-test trials. An analysis of serial position curves for jumps of varying sizes and directions in the spin list suggests that the flattening results from the movement of the prior trial's primacy items to other positions within the list. Larger jumps were associated with both increased item gains and increased item losses as compared with smaller jumps ( $p s<.05$ for both effects).

(Manuscript received January 16, 2009; revision accepted for publication July 16, 2009.) 